\title{
Use of very long-distance NOEs in a fully deuterated protein: an approach for rapid protein fold determination
}

\author{
Leonardus M.I. Koharudin, Alexandre M.J.J. Bonvin, Robert Kaptein, and Rolf Boelens* \\ Bijvoet Center for Biomolecular Research, NMR Spectroscopy, Utrecht University, Padualaan 8, 3584 CH, Utrecht, The Netherlands
}

Received 27 December 2002; revised 18 April 2003

\begin{abstract}
The high sensitivity of modern NMR instrumentation, in combination with full deuteration, enabled the measurement of longrange NOEs between amide protons in a fully deuterated protein corresponding to distances up to $8 \AA$. These are beyond the limit normally observed in protonated samples. Such long-distance NOEs could be observed using long mixing times, which became possible due to reduced spin diffusion and $T_{1}$ relaxation of the amide protons in the fully deuterated sample. This information was used in combination with secondary structure restraints derived from secondary chemical shifts for structure calculations. With these backbone amide proton NOEs only, a unique fold could be obtained with positional root mean square deviations from the average of 1.30 and $2.25 \AA$ for backbone and heavy atoms, respectively. Despite the low density of restraints, no mirror image problems were observed. Addition of sidechain NOE information increased the precision of the ensemble and in particular of the core packing. The structures obtained in this way were close to the published crystal structure. NOE completeness analysis revealed that the cumulative completeness is still more than $80 \%$ for an $8.0 \AA$ cut-off distance.
\end{abstract}

(C) 2003 Elsevier Science (USA). All rights reserved.

Keywords: CI2; Deuteration; NMR; NOE

\section{Introduction}

The reduction in dipolar proton-proton relaxation in partially deuterated proteins can be used to improve both the resolution and the sensitivity of homonuclear and heteronuclear NMR experiments [21,22,27,29,33, $37,43,48-50])$. The resulting longer $T_{1}$ relaxation times and the absence of spin diffusion allow the detection of weaker NOEs when significantly longer mixing times are used than what is common practice $[14,26,41]$. The resulting very long distances between amide protons in fully deuterated proteins can be used as the main source of structural information for the fold determination $[26,42,44]$. The advantage of such an approach is that a reliable protein fold can be generated at an early stage of the NMR structure determination process, simplifying further spectral analysis. Thus far, however, the NMR sensitivity has been too low in practice to observe a sufficiently large set of long-range distances. This ap-

\footnotetext{
${ }^{*}$ Corresponding author. Fax: +31-30-253-7623.

E-mail address: boelens@nmr.chem.uu.nl (R. Boelens).
}

proach generated at best structures of low resolution. Additional long-range distance information was required such as obtained by selective protonation of methyl or aromatic groups $[8,9,11,31,32,35,39]$. This adds, however, an additional complication, not only biochemically, but also in the NMR analysis with the need of resonance assignment of methyl or aromatic groups.

Current high-field instruments and cryoprobe technology have improved significantly the sensitivity of NMR as compared to 5 years ago. In addition, reliable NMR structure calculation protocols have been developed with improved convergence properties. Distance information from only amide protons can be combined with secondary structure restraints derived from ${ }^{13} \mathrm{C}$, ${ }^{15} \mathrm{~N}$, and ${ }^{1} \mathrm{H}$ backbone chemical shift analysis [5,47]. This information can be supplemented with additional NMR parameters such as residual dipolar coupling constants from aligned molecules [40] or cross-hydrogen bond J-couplings [13]. The resulting structural information might, however, not always be sufficient to uniquely define the three-dimensional structure. The real 
challenge is therefore to obtain a set of long-distance NOEs that can uniquely define the relative position of the secondary structure elements with respect to each other. In $\beta$-sheets long-range $d_{N N}$ distance restraints between the extended strands (typically $=2.6 \AA$ ) can be easily observed, even in fully protonated samples. Longrange $d_{N N}$ distances of about $6 \AA$ are required to properly pack $\alpha$-helices against $\beta$-sheets, whereas distances of 7-8 $\AA$ are needed to pack two $\alpha$-helices. Mal et al. [26] could observe NOEs up to $6.8 \AA$ in the ${ }^{15} \mathrm{~N}-,{ }^{2} \mathrm{H}$-labeled Fyn protein. By using NOE information from both backbone and sidechain tryptophan $\mathrm{NH}$ protons supplemented with 35 additional ${ }^{3} \mathrm{~J}\left(\mathrm{H}_{\mathrm{N}}, \mathrm{H}_{\alpha}\right)$ torsion angle restraints the NMR structure of this all $\beta$ protein could be obtained. The relative low number of restraints caused, however, convergence and precision problems and partial mirror images could not be avoided. These problems, even with NOEs corresponding to longer distances (up to $\sim 7.0 \AA$ ) than what is commonly used, indicate that either sidechain information is needed, e.g., by selectively protonating methyl groups, or alternatively, that even longer distance NOEs should be measured that can uniquely define the fold of a protein. This is particularly true for the more general case of mixed $\alpha / \beta$-proteins, but even more so for all $\alpha$-helical proteins.

This paper reports the observation of very long-distance NOEs up to $8.0 \AA$ between amide protons of different structural elements of a fully deuterated protein. Such long-distance NOEs in the absence of spin diffusion have thus far not been reported. We show that the long-distance NOE information obtained, supplemented with dihedral angle restraints that can be derived from ${ }^{13} \mathrm{C},{ }^{15} \mathrm{~N}$, and ${ }^{1} \mathrm{H}_{\mathrm{N}}$ secondary chemical shift information, is sufficient to obtain a reliable three-dimensional model. This methodology has been applied to the chymotrypsin inhibitor type 2 (CI2), a small, compact, $\alpha / \beta$ protein that consists of 64 amino acids [46] for which both crystal [30] and solution [24] structures have already been determined, allowing the assessment of the precision and accuracy of this approach.

\section{Materials and methods}

\subsection{Expression and purification of deuterated CI2}

Deuteration was accomplished following the scheme reported previously by Gardner and Kay [10]. In order to fully deuterate $\mathrm{CI} 2$, however, the random deuteration scheme was modified and ${ }^{2} \mathrm{H} /{ }^{13} \mathrm{C}$ glucose was used as the carbon source to ensure high deuteration incorporation [20]. The CI2 gene without the first 19 unstructured residues was encoded in a pJM-CI2 plasmid that was previously transformed into Escherichia coli BL21 competent cells [16]. The cells were first grown in M9 minimal medium in $\mathrm{H}_{2} \mathrm{O}$ and then transferred to the
$\mathrm{D}_{2} \mathrm{O}$ medium by centrifugation of the cell culture and resuspension in the new medium. This transfer step was repeated twice. No adaptation to the deuterated medium was needed. For the final culture, $1.25 \mathrm{~L}$ of the cell culture was induced with $1.0 \mathrm{mM}$ IPTG at an $\mathrm{OD}_{600}$ of $\sim 0.7$. The cells were harvested $8 \mathrm{~h}$ after induction. $\mathrm{Pu}-$ rification of the ${ }^{2} \mathrm{H}-/{ }^{13} \mathrm{C}-/{ }^{15} \mathrm{~N}$-labeled $\mathrm{CI} 2$ was performed as described in by Jackson and Fersht [16].

Although the cell growth rate in the $\mathrm{M} 9 / \mathrm{D}_{2} \mathrm{O}$ medium was $50 \%$ slower than in $\mathrm{M} 9 / \mathrm{H}_{2} \mathrm{O}$ minimal media, the final expression yields were comparable. The yield, after purification, was $\sim 35 \mathrm{mg}$ of dried protein per liter of culture. Comparison of the decoupled and undecoupled 1D ${ }^{1} \mathrm{H}$ and ${ }^{13} \mathrm{C}$ NMR spectra and MALDI-TOF mass spectrometry indicated an incorporation of ${ }^{2} \mathrm{H},{ }^{15} \mathrm{~N}$, and ${ }^{13} \mathrm{C}$ isotopes of 97,99 , and $98 \%$, respectively.

\subsection{NMR experiments and chemical shift analysis}

${ }^{2} \mathrm{H}-/{ }^{13} \mathrm{C}-/{ }^{15} \mathrm{~N}-$ Labeled CI 2 protein $(7.33 \mathrm{mg})$ was dissolved in $0.65 \mathrm{ml}$ of $50 \mathrm{mM} \mathrm{NaAc} / \mathrm{HAc}$ buffer, $\mathrm{pH}$ 4.4, containing $0.02 \%$ sodium azide and $5 \% \mathrm{D}_{2} \mathrm{O}$. All NMR experiments $\left(1 \mathrm{D}{ }^{1} \mathrm{H}, 2 \mathrm{D}{ }^{15} \mathrm{~N}-{ }^{1} \mathrm{H}\right.$ HSQC, 3D HNCA, 3D HN(CO)CA, 3D HNCO, 3D HN(CA)CO, $3 \mathrm{D} \mathrm{HNCACB}$, and $3 \mathrm{D} \mathrm{HN}(\mathrm{CO}) \mathrm{CACB}$, essentially as described in [4]) were performed at $300 \mathrm{~K}$ on a four channel Bruker DRX600 spectrometer. NMRPipe [6] was used for NMR data processing. Assignments of ${ }^{1} \mathrm{H}_{\mathrm{N}},{ }^{15} \mathrm{~N}$, ${ }^{13} \mathrm{C}_{\alpha},{ }^{13} \mathrm{C}_{\beta}$, and ${ }^{13} \mathrm{C}^{\prime}$ chemical shifts of the deuterated protein were carried out with NMRView [17]. The chemical shifts of the sidechain ${ }^{1} \mathrm{H}_{\mathrm{N}}$ and ${ }^{15} \mathrm{~N}$ resonances were obtained from the previous assignments of the protonated sample [1] (BMRB Accession Code 4974).

\subsection{NOE rate analysis and NOE intensity calibration}

Two-dimensional NOE build-up experiments were acquired with 16 scans at $300 \mathrm{~K}$ on a four channel Bruker DRX600 spectrometer. The 2D NOE spectrum used for the final collection of the amide proton NOEs was recorded at $300 \mathrm{~K}$ on a four channel Bruker DRX750 spectrometer with a $1600 \mathrm{~ms}$ mixing time, 96 scans, $1024 \times 512$ complex points on $F 1 \times F 2$ and a recycling delay of $4 \mathrm{~s}$.

Although the distance restraints were automatically calibrated by ARIA, a manual calibration of NOE crosspeak intensities was carried out in order to define the maximum cut-off for the ARIA automated calibration procedure, using the equation $\left.d_{i j}=\left(1 / d_{\text {ref }}\right)\left({ }^{6} \sqrt{(} I_{\text {ref }} / I_{i j}\right)\right)$ (where $I_{i j}$ is the intensity of the cross-peak between the $i$ and $j$ spins, $d_{\text {ref }}$ is a reference distance, and $I_{\text {ref }}$ is the corresponding intensity). Two references were used for this purpose: the average $\mathrm{H}_{N}-\mathrm{H}_{N+1}$ NOE intensity in the $\alpha$-helical region corresponding to a distance of $2.8 \AA$ and the average $\mathrm{H}_{N}-\mathrm{H}_{N+1} \mathrm{NOE}$ intensity in the $\beta$-sheet region corresponding to a distance of $4.4 \mathrm{~A}$. The 
corresponding distances between backbone amide protons in CI2 were measured from the crystal structure (2CI2) after addition of the hydrogen atoms with SPDBViewer [15].

\subsection{Structure calculations}

The NMR structures were calculated with ARIA [34] and CNS [3] using the Parallhdg5.2 force field with the PROLSQ parameters [23] as described in Bonvin et al. [1]. The distance restraints were automatically calibrated by ARIA. Considering both the analysis of weak NOE crosspeaks against the corresponding distances in the crystal structure and the manual calibration, the upper distance cut-off for calibration in the calibrate.inp file was set to $8.0 \AA$. Eight consecutive iterations were carried out and 200 structures were calculated in each iteration. The 50 structures with lowest restraint energy were finally refined in explicit water using the OPLS parameters [18]. The selection of the final structures to represent the ensemble was based on the profile of maximum backbone RMSD from the average (residues 3-62) against increasing ensemble sizes according to restraint energy sorted structures. In addition, the structures that contained NOE violations $>0.3 \AA$ or dihedral angle violations $>5^{\circ}$ were rejected. After selection, the final structures making up each ensemble were analyzed with PROCHECK-NMR [19], PROSAII [38], and Profiler3D [2,25]. All RMSD calculations were done with the Profit program [28].

\section{Results}

Comparison of the carbon chemical shifts between the protonated and deuterated CI2 samples revealed that, in general, every replacement of a proton with a deuteron shifted the resonances of the attached carbon by $\sim 0.4 \mathrm{ppm}$ upfield, in agreement with what has been previously reported [10,12]. After chemical shifts adjustment to account for the deuterium isotope labeling, the 64 residues of $\mathrm{CI} 2$ for which chemical shift information for ${ }^{13} \mathrm{C}_{\alpha},{ }^{13} \mathrm{C}_{\beta},{ }^{13} \mathrm{C}^{\prime}$, and ${ }^{15} \mathrm{~N}$ atoms was available (for the four prolines, no ${ }^{15} \mathrm{~N}$ assignments were available) were analyzed with the TALOS program [5] in a conservative manner as described before [1]. The TALOS analysis resulted in 39 good predictions of $\phi$ and $\psi$ dihedral angles, corresponding to $60 \%$ of the 64 residues analyzed. Most of these predictions are for residues that are involved in secondary structural elements such as residues $13-24$ in the $\alpha$-helix region and residues $28-34$, $46-49,51,53,54,60,62$, and 63 in the $\beta$-sheet regions.

\subsection{NOE analysis}

The optimum NOE mixing time for the deuterated CI2 protein was obtained from a rate analysis of $2 \mathrm{D}$ NOE experiments recorded at five different mixing times ranging from 100 to $2400 \mathrm{~ms}$. Fig. 1 shows cross-sections of $2 \mathrm{D}$ NOE spectra corresponding to the backbone amide proton of $\mathrm{E} 7$ and the sidechain NH proton of W5 at different mixing times. Four and ten NOEs were observed for E7 and W5, respectively. The corresponding distances in the crystal structure are indicated in the figure. Distances up to $\sim 4.4 \AA$ were readily observed with a mixing time of $100 \mathrm{~ms}$. Longer distances became only observable at mixing times of $200-400 \mathrm{~ms}$. All cross-peaks reached their maximum intensity at a mixing time of about $1600 \mathrm{~ms}$ and started to decrease after this point due to $T_{1}$ relaxation. A similar behavior could be observed for most cross-peaks present in the $2 \mathrm{D}$

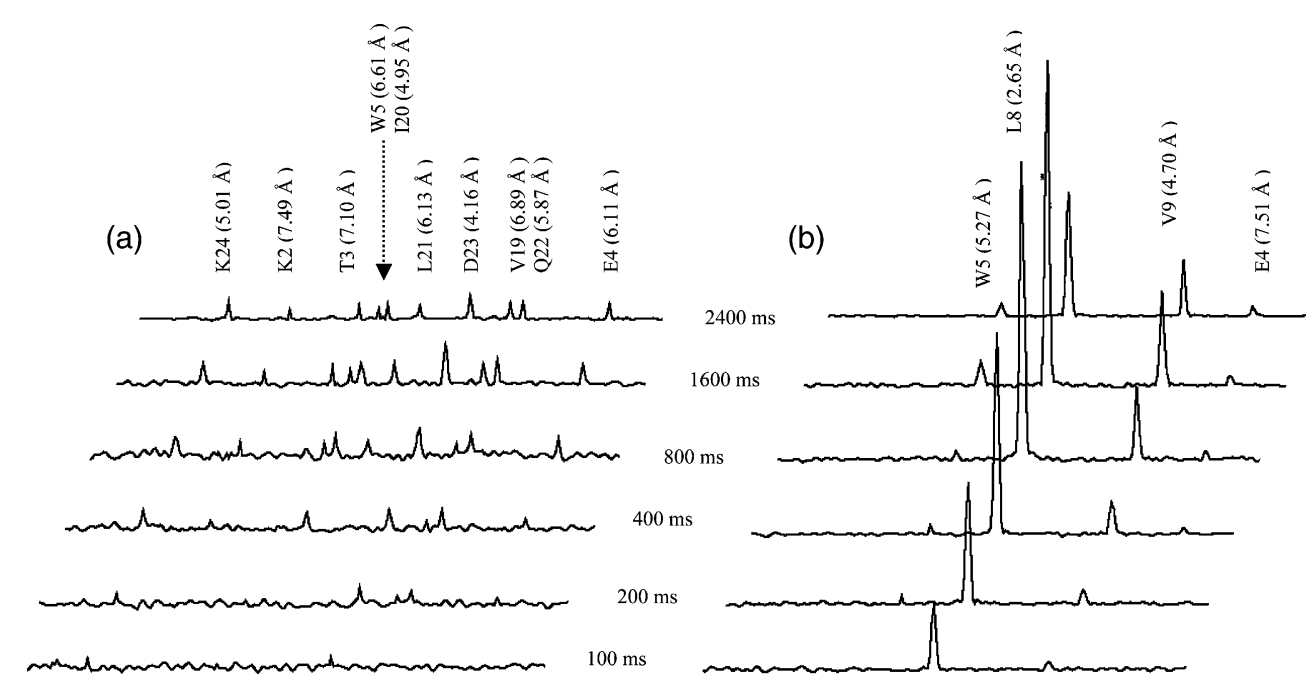

Fig. 1. Cross-sections from 2D homonuclear NOESY datasets recorded at $600 \mathrm{MHz}$ showing the build-up of the inter amide proton NOEs as a function of mixing time. The data are for the $\mathrm{H}_{\mathrm{N} \varepsilon}$ of W5 (a) and the backbone $\mathrm{H}_{\mathrm{N}}$ of glutamate $\mathrm{E} 7$ (b). The peak assignments and the corresponding distances (between brackets) in the crystal structure (2CI2) are indicated on top. 


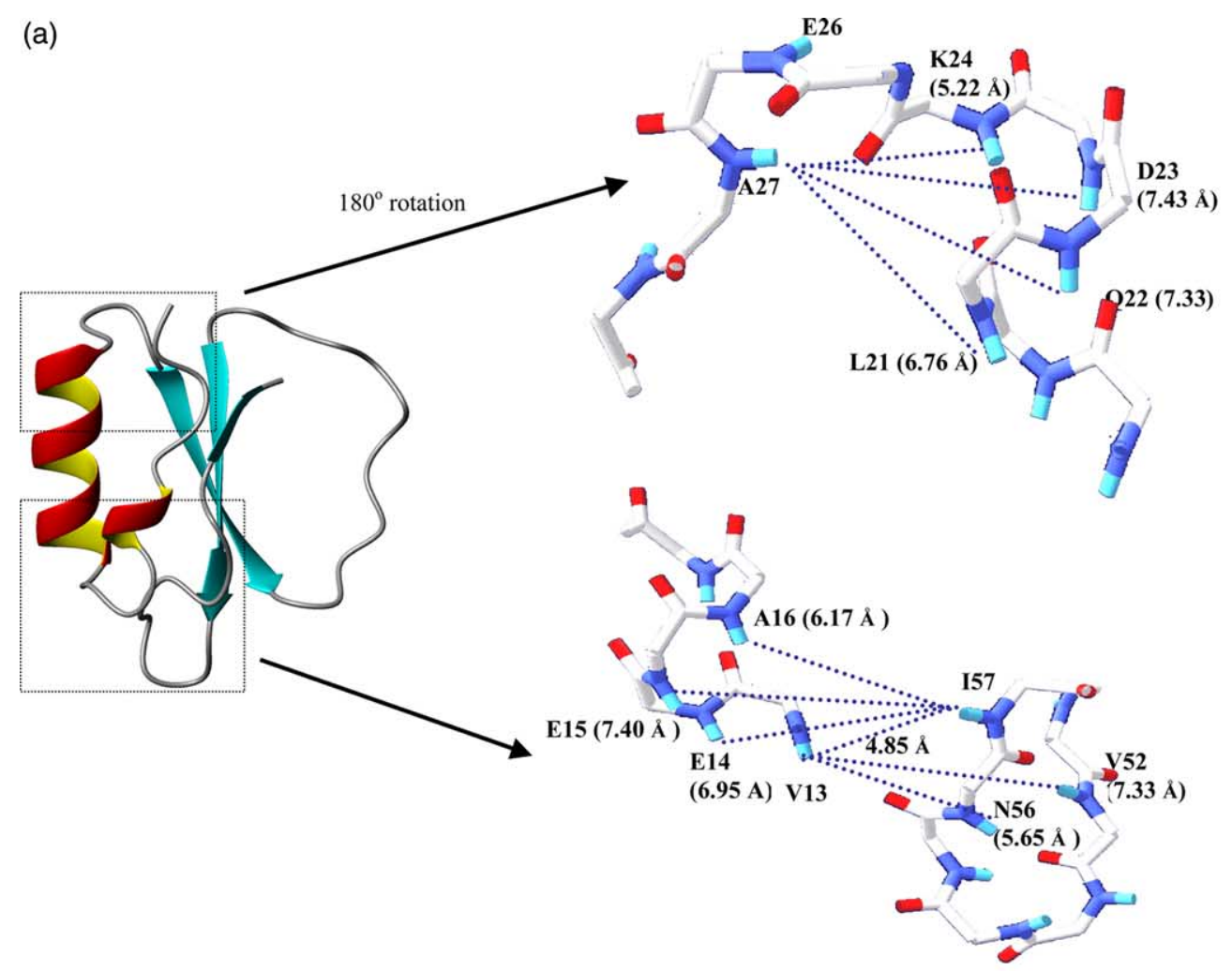

(b)

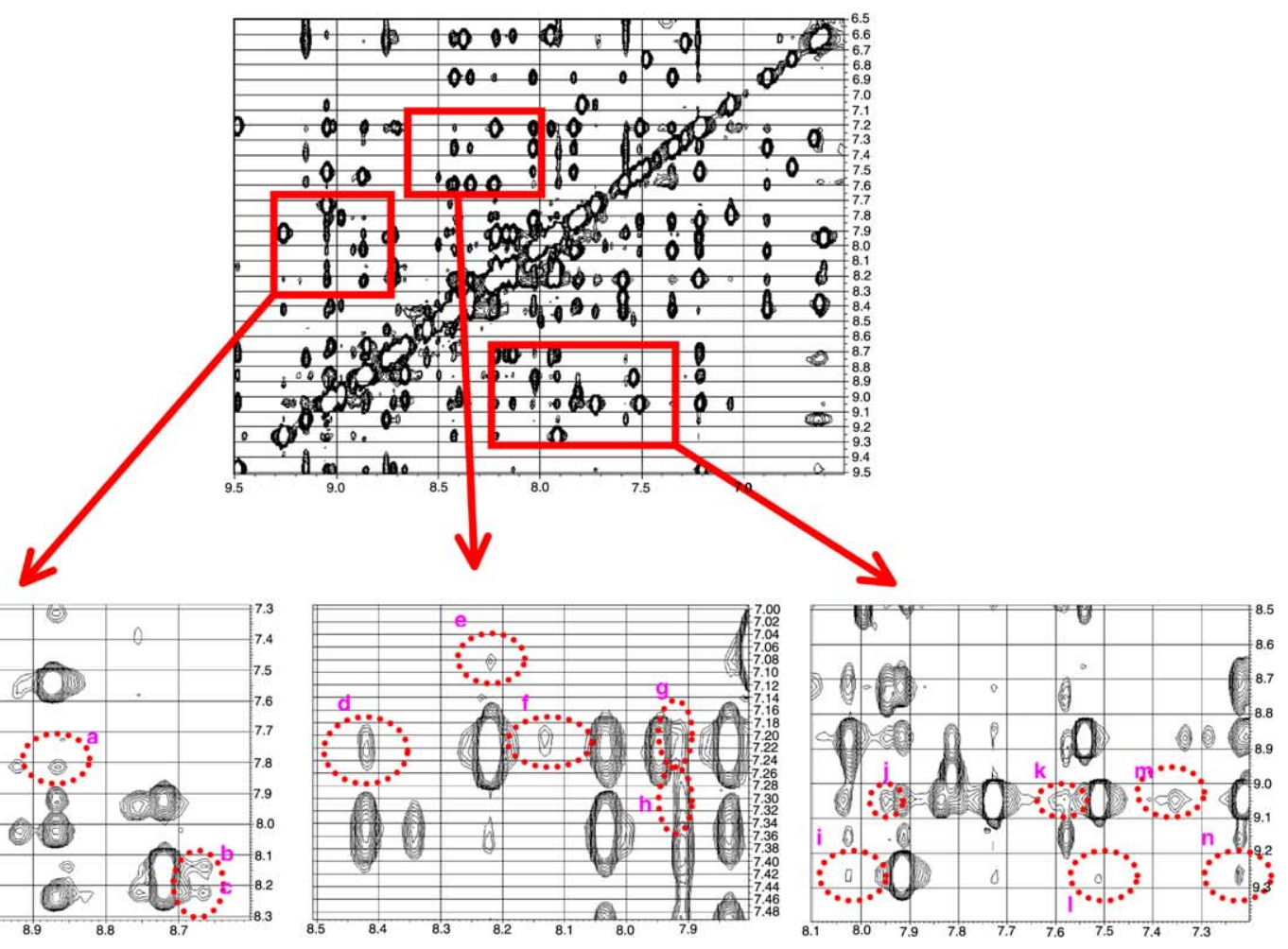

Fig. 2. Representative long-distance NOEs of CI2. (a) Crystal structure (PDB 2CI2) with observed NOEs for Ala27 (top box) and Ile57 (bottom box). The distances between brackets were measured in the crystal structure. (b) $2 \mathrm{D}$ NOE spectrum of $1.5 \mathrm{mM}{ }^{2} \mathrm{H}-,{ }^{15} \mathrm{~N}$-, and ${ }^{13} \mathrm{C}-\mathrm{labeled} \mathrm{CI} 2$ recorded at $750 \mathrm{MHz}$ with a mixing time of $1600 \mathrm{~ms}$. The labels a to $\mathrm{n}$ indicate the following long-range NOEs (with the distance in the X-ray structure between brackets): a, Leu8-Val60 (7.33 $)$; b, Val51-Lys53 (7.66 $)$; c, Val51-Asp55 (7.06 $)$; d, Lys17-Gln22 (7.85 $)$; e, Trp5-Val9

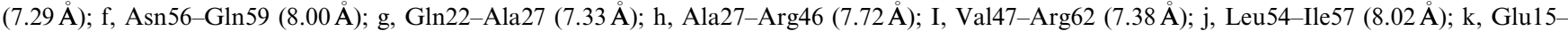
Ile57 (7.40 ̊); 1, Asp23-Glu26 (7.54 $)$ ); m, Val19-Lys24 (7.71 А); n, Gln22-Glu26 (7.87 $\AA$ ). The intensities of these NOEs were calibrated by Aria into upperbounds of $7.5 \AA$. 


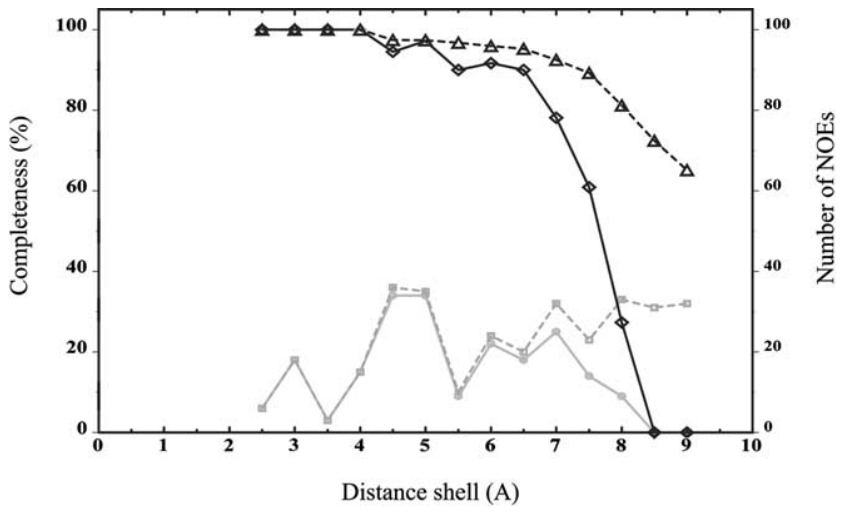

Fig. 3. NOE completeness (in \%) versus distance shell for deuterated CI2: dashed black line, cumulative completeness; solid black line, completeness per unit shell; solid gray line, number of observed NOEs per unit shell; and dashed gray line, number of expected NOEs per unit shell. The thickness of the shell is $0.5 \AA$.
NOE spectrum. The optimum mixing time of $1600 \mathrm{~ms}$ for the NOESY experiment corresponds to $82 \%$ of the average $T_{1}$ value of the deuterated CI2 protein ( $T_{1}$ data not shown).

The 2D NOE experiment with $1600 \mathrm{~ms}$ mixing time on a conventional $600 \mathrm{MHz}$ NMR spectrometer resulted in observable cross-peaks for distances up to $7 \AA$. In this spectrum, the sequential $d_{N N(i, i+1)}$ connectivities are very intense and, in most cases, the $d_{N N(i, i+2)}$ and $d_{N N(i, i+3)}$ connectivities that have been reported previously were also clearly visible $[14,41]$. These $7 \AA$ NOEs alone were sufficient to uniquely define the conformation of the $\beta$ sheet of CI2. However, mirror image problems were still present, since the position of the helix was not uniquely defined: the $7 \AA$ distances allowed the positioning of the helix either above or below the plane defined by the $\beta$ sheets. Similar mirror image problems were reported previously when using a limited number of NOEs [26] or cross-hydrogen-bond coupling information only [1].

Table 1

Summary of input and structural statistics

\begin{tabular}{|c|c|c|}
\hline Input statistics & RunA & RunB \\
\hline \multicolumn{3}{|l|}{ Distance restraints } \\
\hline Backbone & 189 & 189 \\
\hline Side chain NOEs & - & 30 \\
\hline Dihedral angle restraints & 78 & 78 \\
\hline Total restraints & 267 & 297 \\
\hline Structural statistics & 41 Structures & 42 Structures \\
\hline \multicolumn{3}{|l|}{ Ramachandran plot } \\
\hline Most favored regions (\%) & 80.5 & 80.9 \\
\hline Additional allowed regions $(\%)$ & 16.2 & 17.0 \\
\hline Generously allowed regions (\%) & 2.2 & 1.4 \\
\hline Disallowed regions (\%) & 1.1 & 0.7 \\
\hline \multicolumn{3}{|l|}{ RMSD for experimental restraints ${ }^{\mathrm{a}}$} \\
\hline Distance restraints $(\AA)$ & $0.022 \pm 0.003$ & $0.026 \pm 0.003$ \\
\hline Dihedral angle restraints $\left({ }^{\circ}\right)$ & $0.79 \pm 0.18$ & $0.73 \pm 0.18$ \\
\hline \multicolumn{3}{|l|}{ CNS energies after water refinement ${ }^{b}$} \\
\hline$E_{\mathrm{vdw}}\left(\mathrm{kcal} \mathrm{mol}^{-1}\right)$ & $-610 \pm 13$ & $-615 \pm 11$ \\
\hline$E_{\text {elec }}\left(\mathrm{kcal} \mathrm{mol}^{-1}\right)$ & $-2778 \pm 114$ & $-2916 \pm 90$ \\
\hline \multicolumn{3}{|l|}{ RMSD from idealized covalent geometry } \\
\hline Bonds $(\AA)$ & $0.0038 \pm 0.0002$ & $0.0040 \pm 0.0001$ \\
\hline Angles $\left({ }^{\circ}\right)$ & $0.45 \pm 0.03$ & $0.46 \pm 0.02$ \\
\hline Impropers $\left(^{\circ}\right)$ & $0.46 \pm 0.05$ & $0.47 \pm 0.04$ \\
\hline Profiler3D $z$-score ${ }^{\mathrm{c}}$ & $11.7 \pm 3.0$ & $10.3 \pm 2.1$ \\
\hline Prosa II $z$-score ${ }^{\mathrm{c}}$ & $-5.45 \pm 0.25$ & $-5.45 \pm 0.25$ \\
\hline \multicolumn{3}{|l|}{ Average RMSD to mean $(\AA)^{d}$} \\
\hline All residues (backbone/heavy atoms) & $1.30 \pm 0.30 / 2.25 \pm 0.30$ & $0.85 \pm 0.15 / 1.70 \pm 0.20$ \\
\hline Secondary elements (backbone/heavy atoms) & $0.70 \pm 0.25 / 1.50 \pm 0.20$ & $0.55 \pm 0.10 / 1.30 \pm 0.15$ \\
\hline \multicolumn{3}{|c|}{ Average RMSD $(\AA)$ in ensembles against X-ray (2CI2) } \\
\hline All residues (backbone/heavy atoms) & $1.75 \pm 0.35 / 3.00 \pm 0.40$ & $1.30 \pm 0.15 / 2.50 \pm 0.20$ \\
\hline Secondary elements (backbone/heavy atoms) & $0.95 \pm 0.20 / 2.15 \pm 0.20$ & $0.85 \pm 0.10 / 2.05 \pm 0.15$ \\
\hline \multicolumn{3}{|c|}{ RMSD (£) of the representative structure from each ensemble to X-ray (2CI2) } \\
\hline All residues (backbone/heavy atoms) & $1.05 / 1.95$ & $1.05 / 2.20$ \\
\hline Secondary elements (backbone/heavy atoms) & $0.85 / 1.75$ & $0.75 / 1.95$ \\
\hline
\end{tabular}

${ }^{a}$ No distances restraint violations $>0.3 \AA$ and no dihedral angle restraint violations $>5^{\circ}$.

${ }^{\mathrm{b}}$ The non-bonded energies were calculated with the OPLS parameters using an $8.5 \AA$ cut-off.

${ }^{\mathrm{c}}$ Crystal structure (2CI2) profiler3D $z$-score is 9.87 and prosaII $z$-score is -6.06 .

d All residues: 3-64 and secondary structure residues: 13-24, 28-33, 45-52, 60-62 (result from TALOS prediction). 
Although the correct fold can be identified by various methods, see example Bonvin et al. [1], a unique fold is preferable.

After recording a 2D NOE experiment with the same mixing time $(1600 \mathrm{~ms})$ at $750 \mathrm{MHz}$ with 96 scans, new important long-distance NOEs could be observed. Some of these are shown in Fig. 2. In retrospect, these longdistance NOEs were already present in the $600 \mathrm{MHz} 2 \mathrm{D}$ NOE spectrum with 16 scans, but their intensity was weak and close to the noise level. The fivefold increase in number of scans and the use of the $750 \mathrm{MHz}$ spectrometer brought up these cross-peaks to intensities that are relatively easy to discriminate from the noise. As described in Section 2, we estimated that the maximum distances that would be observable in the $750 \mathrm{MHz}$ spectra of CI2 would be $8.0 \AA$. This was confirmed by comparing weak NOE cross-peaks with the corresponding distances in the CI2 crystal structure (indicated between brackets in Fig. 2). There is a good correspondence between the distances derived from the NOE calibration procedure (see Section 2) and the distances in the crystal structure since there are no intervening protons that could contribute to spin-diffusion between the secondary structure elements (data not shown). Such long-distance NOEs, up to $8.0 \AA$, have not been described previously for protonated protein samples. Most importantly, many of these long-distance cross-peaks originate from protons in different secondary structure elements of CI2, such as from the amide protons at the end of the $\alpha$-helix to $\beta$ sheets and from the amide protons at the beginning of the $\alpha$-helix to the loop region (Fig. 2). These NOEs are particularly important because they define the position of the $\alpha$-helix with respect to the $\beta$ sheet and thus remove any mirror image problems.

We also analyzed the experimental NOE data in terms of NOE completeness [7,45] using the crystal structure (2CI2) (Fig. 3). The NOE completeness is defined as the ratio between the number of observed NOEs and the number of expected NOEs based on the threedimensional structure. Since the protein is fully deuterated, only amide protons were considered. The decrease in the number of observed NOEs after the $6.5 \AA$ cut-off shell is due to either spectral overlap or too weak intensity. The lower and medium distance shells (under $5.5 \AA$ ) have approximately $93 \%$ NOE completeness. The NOE completeness for the $7.5-8.0 \AA$ shell is just over $20 \%$, whereas the cumulative completeness, i.e., up to a certain cut-off distance, is still more than $80 \%$ at $8.0 \AA$. No NOEs were found corresponding to distances above $8.0 \AA$.

\subsection{Use of long-distance NOEs in structure calculation}

Two independent sets of structure calculations, denoted runA and runB, were performed using two sets of structural restraints extracted from the $2 \mathrm{D}$ NOE ex- periments, complemented by dihedral angle restraints derived from the secondary chemical shift analysis with TALOS [5]. Two hundred and sixty-nine NOE crosspeaks resulting in 189 distance restraints and 78 dihedral angles were used in runA while 30 additional distance restraints derived from 56 cross-peaks involving sidechain NH protons of tryptophan, glutamine, asparagine, and arginine were used in runB (Table 1). The protocols were in both cases the same. From the 50 water refined structures, 41 and 42 structures without any NOE violations were obtained for runA and runB, respectively, after analysis (see Section 2). In both cases, a unique fold was obtained. Fig. 4 shows the per residue RMSD plot for both sets of calculated structures. It can clearly be seen that the first few residues and the three loop regions, i.e., residues 25-26, 36-44, and 53-56, have higher RMSDs than the rest of the sequence, which can be explained by the lack of long-distance NOEs in those parts. Some regions converged significantly better after including sidechain NOE information, especially the first few residues and the long loop (residues 36-44).

The structural statistics for both runs are summarized in Table 1. Despite the limited numbers of NOEs, a high percentage of the residues fall into the most favorable

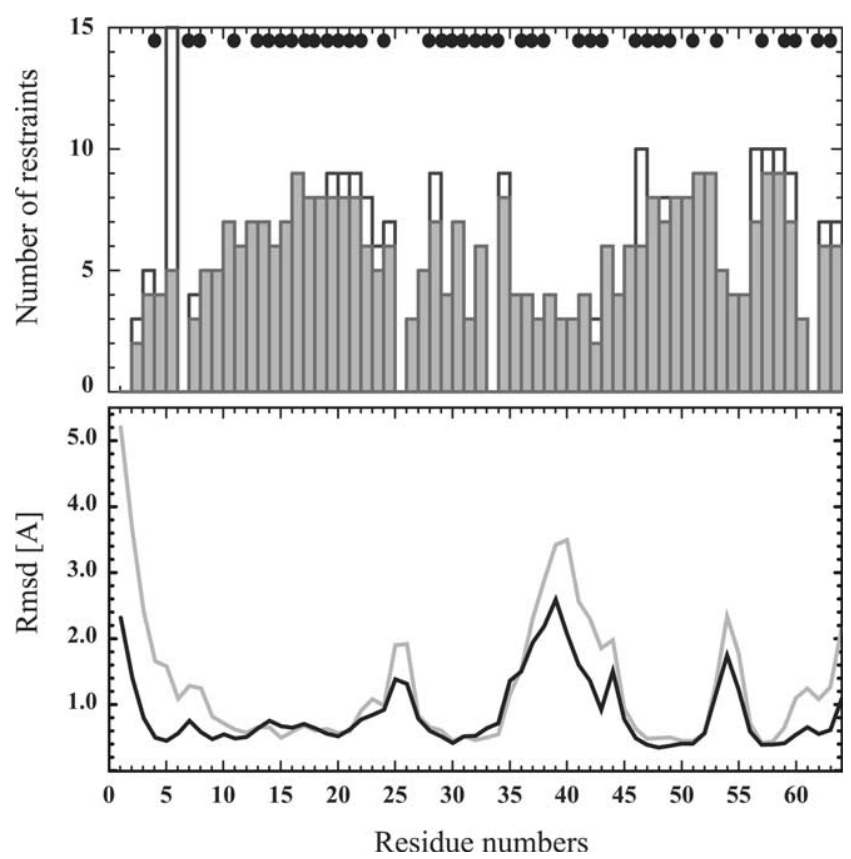

Fig. 4. Number of restraints and sequential RMSD, as a function of the residue sequence for the two ensembles of structures calculated with backbone proton NOEs and with backbone and sidechain proton NOEs. The number of backbone proton NOEs is shown as filled gray bars and sidechain proton NOEs as open bars. The black circles on top of the NOE restraints indicate the residues for which dihedral angle restraints were predicted with TALOS [5]. In the sequential RMSD graph, the results from the structure calculations using only backbone proton NOEs are indicated in gray and the ones with additional sidechain proton NOEs in black. The structures were superimposed on $\mathrm{C} \alpha, \mathrm{C}$, and $\mathrm{N}$ atoms of residue 3-62. 

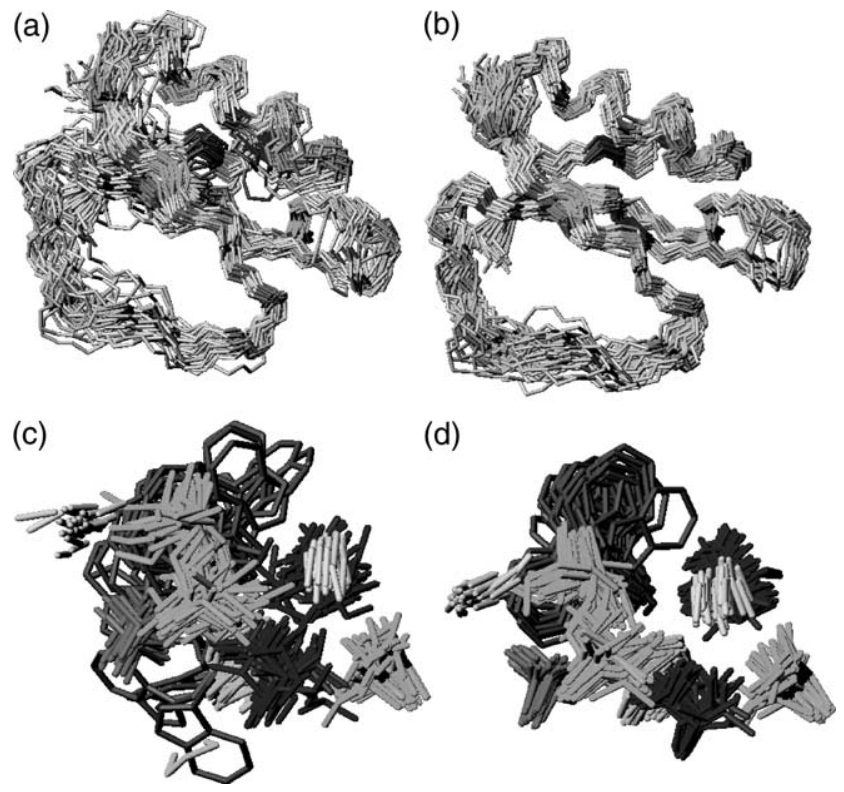

Fig. 5. Backbone representation and core packing of the CI2 structures calculated from backbone amide proton NOEs only (a)/(c) and with inclusion of the 30 sidechain proton NOEs $(\mathrm{b}) /(\mathrm{d})$. Core residues are indicated in dark gray.

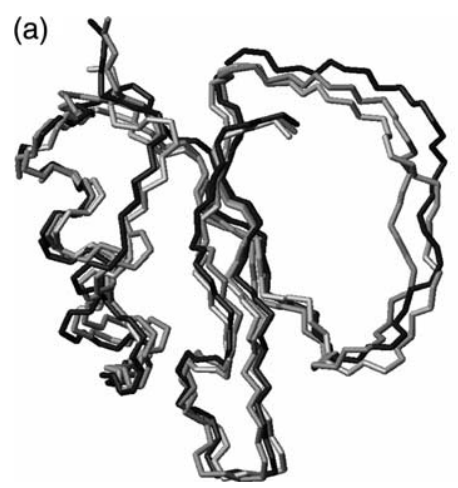

(b)

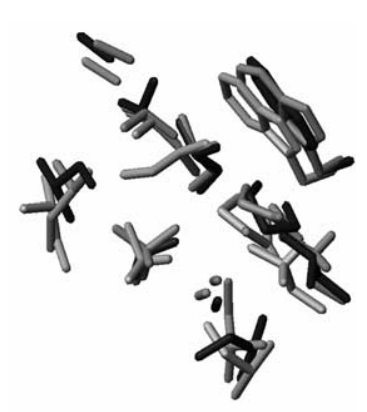

Fig. 6. Superposition of the representative structure of ensemble (runA, dark gray; runB, light gray) and the crystal structure (2CI2) (black). The structures were superimposed on backbone atoms of residues 3-62. (a) Backbone, (b) core residues. The representative structures were taken as the closest to the average of each ensemble.

regions of the Ramachandran plot: 80.5 and $80.9 \%$ for runA and runB, respectively, and 96.7 and $97.9 \%$. when including additionally allowed regions. These values are comparable to the $98.2 \%$ calculated for the crystal structure (2CI2). The ProsaII [38] and Profiler_3D [2] scores of runA and runB (Table 1) are also close to those of the crystal structure (-6.06 and 9.87 for ProsalI and Profiler_3D, respectively).

The two ensembles of structures are depicted in Fig. 5 and show a good overlay. The ensemble RMSD to the average is $1.30 \pm 0.30$ and $2.25 \pm 0.30 \AA$ for backbone and heavy atoms of residues 3-64 in runA. The addition of sidechain NOEs in runB reduced the corresponding
RMSD values to $0.85 \pm 0.15$ and $1.70 \pm 0.20 \AA$ and had an even larger impact on the precision of the sidechains of core residues as depicted in Figs. $5 \mathrm{c}$ and d. The ensemble precision in run $\mathrm{B}$ is surprisingly comparable to the one of the published solution structure of CI2 [24] which was calculated using a much larger number of restraints (RMSD to the average for backbone and heavy atoms of $0.8 \pm 0.2$ and $1.6 \pm 0.2 \AA$, respectively).

Both sets of structures were compared to the crystal structure (Table 1). The structures from runA are already quite similar to the crystal structure with an average backbone RMSD from the crystal of $1.75 \pm 0.35 \AA$ and of only $0.95 \pm 0.20 \AA$ when only secondary structure elements are considered. These values drop to $1.30 \pm 0.15$ and $0.85 \pm 0.10 \AA$ when sidechain information is included (runB). A similar trend is observed for the heavy atom RMSDs. The best defined ensemble (runB) is also the closest to the crystal structure. Fig. 6 shows a superimposition of the representative structures (closest to the average) from the two runs and of the crystal structure. The RMSDs over the secondary elements of the representative structures from the crystal structure are as low as 0.85 and $0.75 \AA$ for run $A$ and runB, respectively. Considering the relatively limited numbers of restraints, the quality of the structures from both sets of calculations is quite good.

\section{Discussion}

Complete replacement of all non-exchangeable protons by deuterium is extremely useful for a rapid backbone assignment since all resonances are characterized by long $T_{2}$ relaxation times and sharp signals. Here we have shown that the same sample can also be useful for structure determination. The long $T_{1}$ relaxation times of the amide protons allow the use of very long-NOE mixing time so that weak NOEs can be measured. In addition, the low proton density reduces the risk of spin diffusion. Using this approach, we could detect, for the first time, NOEs corresponding to distances up to $\sim 8.0 \AA$.

Further sensitivity improvements can be achieved with cryoprobes and higher field instruments. An estimate indicates that a signal-to-noise improvement of a factor five is feasible in the coming years. This could lead to the observation of almost all long distances up to $\sim 9.0 \AA$ and many additional ones up to $\sim 10.5 \AA$, doubling approximately the number of NOE restraints that we have used here (on average three per residues) and should result in significantly better defined ensembles. This, together with the improvements in data analysis and structure calculations, should make the use of fully deuterated samples extremely valuable, even in the case of relatively small proteins. 
The application of this approach to larger proteins remains a challenge. Next to the increased fold complexity, the protein concentrations will be lower and, although TROSY methods [36] may reduce linewidth problems enormously, it is clear that the sensitivity of the experiments will still be significantly lower. However, in favorable cases, i.e., highly soluble proteins, it can be expected that a similar protocol as outlined above could be successful, increasing the speed of data analysis and structure calculations for these systems possibly by an order of magnitude as well.

\section{Acknowledgments}

The authors would like to thank Dr. A. Fersht and Dr. N.W. Foster for providing pJM-CI2 plasmid and for the protein purification protocols and Ing. A. George for technical advice in setting up the expression and purification systems. This project was funded by the Netherlands Foundation for Chemical Research (NWO/ $\mathrm{CW})$.

\section{References}

[1] A.M. Bonvin, K. Houben, M. Geunneugues, R. Kaptein, R. Boelens, J. Biomol. NMR 21 (2001) 221-233.

[2] J.U. Bowie, R. Luethy, D. Eisenberg, Science 253 (1991) 164-170.

[3] A.T. Brunger, P.D. Adams, G.M. Clore, W.L. DeLano, P. Gros, R.W. Grosse-Kunstleve, J.S. Jiang, J. Kuszewski, M. Nilges, N.S. Pannu, R.J. Read, L.M. Rice, T. Simonson, G.L. Warren, Acta Crystallogr. D Biol. Crystallogr. 54 (1998) 905-921.

[4] J. Cavanagh, W.J. Fairbrother, A.G. Palmer III, N.J. Skelton, in: Protein NMR Spectroscopy: Principle and Practice, Academic press, San Diego, 1996.

[5] G. Corneliscu, F. Delaglio, A. Bax, J. Biomol. NMR 13 (1999) 289-302.

[6] F. Delaglio, S. Grzesiek, G. Vuister, G. Zhu, J. Pfeifer, A. Bax, J. Biomol. NMR 6 (1995) 277-293.

[7] J.F. Doreleijers, M.L. Raves, J.A.C. Rullmann, R. Kaptein, J. Biomol. NMR 14 (1999) 123-132.

[8] K.H. Gardner, M.K. Rosen, L.E. Kay, Biochemistry 36 (1997) 1389-1401.

[9] K.H. Gardner, L.E. Kay, J. Am. Chem. Soc. 119 (1997) 7599-7600.

[10] K.H. Gardner, L.E. Kay, Annu. Rev. Biophys. Biomol. Struct. 27 (1998) 357-406.

[11] N.K. Goto, K.H. Gardner, G.A. Mueller, R.C. Willis, L.E. Kay, J. Biomol. NMR 13 (1999) 369-374.

[12] S. Grzesiek, J. Anglister, H. Ren, A. Bax, J. Am. Chem. Soc. 115 (1993) 4369-4370.

[13] S. Grzesiek, F. Cordier, A.J. Dingley, Methods Enzymol. 338 (2001) 111-133.

[14] S. Grzesiek, P. Wingfield, S. Stahl, J.D. Kaufman, A. Bax, J. Am. Chem. Soc. 117 (1995) 9594-9595.

[15] N. Guex, M.C. Peitsch, Electrophoresis 18 (1997) 2714-2723.
[16] S.E. Jackson, A.R. Fersht, Biochemistry 30 (1991) 10435-10436.

[17] B.A. Johnson, R.A. Blevins, J. Biomol. NMR 4 (1994) 603-614.

[18] W. Jorgensen, J. Tirado-Rives, J. Am. Chem. Soc. 123 (1988) $1541-1542$.

[19] R.A. Laskowski, J.A.C. Rullmann, M.W. MacArthur, R. Kaptein, J.M. Thornton, J. Biomol. NMR 8 (1996) 477-486.

[20] B. Leiting, F. Marsilio, J.F. O’Connell, Anal. Biochem. 265 (1998) 351-355.

[21] D.M. LeMaster, Methods Enzymol. 177 (1989) 23-43.

[22] D.M. LeMaster, F.M. Richards, Biochemistry 27 (1988) 142-150.

[23] J.P. Linge, M. Nilges, J. Biomol. NMR 13 (1999) 51-59.

[24] S. Ludvigsen, H.Y. Shen, M. Kjaer, J.C. Madsen, F.M. Poulsen, J. Mol. Biol. 222 (1991) 621-635.

[25] R. Luethy, J.U. Bowie, D. Eisenberg, Nature 356 (1992) 83-85.

[26] T.K. Mal, S.J. Matthews, H. Kovacs, I.D. Campbell, J. Boyd, J. Biomol. NMR 12 (1998) 259-276.

[27] M.A. Markus, K.T. Kayie, P. Matsudaira, G. Wagner, J. Magn. Reson. B 105 (1994) 192-195.

[28] A.C.R. Martin, 1992. Available from http://www.biochem.ucl.ac. uk/ martin

[29] H. Matsuo, H. Li, G. Wagner, J. Magn. Reson. B 110 (1996) 112 115.

[30] C.A. McPhalen, M.N. James, Biochemistry 26 (1987) 261-269.

[31] A. Medek, E.T. Olejniczak, R.P. Meadows, S.W. Fesik, J. Biomol. NMR 18 (2000) 229-238.

[32] W.J. Metzler, M. Wittekind, V. Goldfarb, L. Mueller, B.T. Farmer II, J. Am. Chem. Soc. 118 (1996) 6800-6801.

[33] D. Nietlispach, R.T. Clowes, R.W. Broadhurst, Y. Ito, J. Keeler, et al., J. Am. Chem. Soc. 118 (1996) 407-415.

[34] M. Nilges, M.J. Macia, S.I. O'Donoghue, H. Osckinat, J. Magn. Reson. B 269 (1997) 408-422.

[35] M.K. Rosen, K.H. Gardner, R.C. Willis, W.E. Parris, T. Pawson, L.E. Kay, J. Mol. Biol. 263 (1996) 627-636.

[36] M. Salzmann, K. Pervushin, G. Wider, H. Senn, K. Wüthrich, PNAS 95 (1998) 13585-13590.

[37] X. Shan, K.H. Gardner, D.R. Muhandiram, N.S. Rao, C.H. Arrowsmith, L.E. Kay, J. Am. Chem. Soc. 118 (1996) 6570-6579.

[38] M.J. Sippl, Proteins 17 (1993) 355-362.

[39] B.O. Smith, Y. Ito, A. Raine, S. Teichmann, L. Ben-Tovim, et al., J. Biomol. NMR 8 (1996) 360-368.

[40] N. Tjandra, J.G. Omichinski, A.M. Gronenborn, A. Bax, Nat. Struct. Biol. 4 (1997) 732-738.

[41] D.A. Torchia, S.W. Sparks, A. Bax, J. Am. Chem. Soc. 110 (1988) 2320-2321.

[42] R.A. Venters, B.T. Farmer II, C.A. Fierke, L.D. Spicer, J. Mol. Biol. 264 (1996) 1101-1116.

[43] R.A. Venters, C.C. Huang, B.T. Farmer II, R. Trolard, L.D. Spicer, C.A. Fierke, J. Biomol. NMR 5 (1995) 339-344.

[44] R.A. Venters, W.J. Metzler, L.D. Spicer, L. Mueller, B.T. Farmer II, J. Am. Chem. Soc. 117 (1995) 9592-9593.

[45] H. Vis, M. Mariani, C.E. Vorgias, K.S. Wilson, R. Kaptein, R. Boelens, J. Mol. Biol. 254 (1995) 692-703.

[46] M.S. Williamson, J. Forde, B. Buxton, M. Kreis, Eur. J. Biochem. 165 (1987) 99-106.

[47] D.S. Wishart, B.D. Sykes, J. Biomol. NMR 4 (1994) 171-180.

[48] T. Yamazaki, W. Lee, C.H. Arrowsmith, D.R. Muhandiram, L.E. Kay, J. Am. Chem. Soc. 116 (1994) 11655-11666.

[49] T. Yamazaki, W. Lee, M. Revington, D.L. Mattiello, F.W. Dahlquist, et al., J. Am. Chem. Soc. 116 (1994) 6464-6465.

[50] T. Yamazaki, H. Tochio, J. Furui, S. Aimoto, Y. Kyogoku, J. Am. Chem. Soc. 119 (1997) 872-880. 